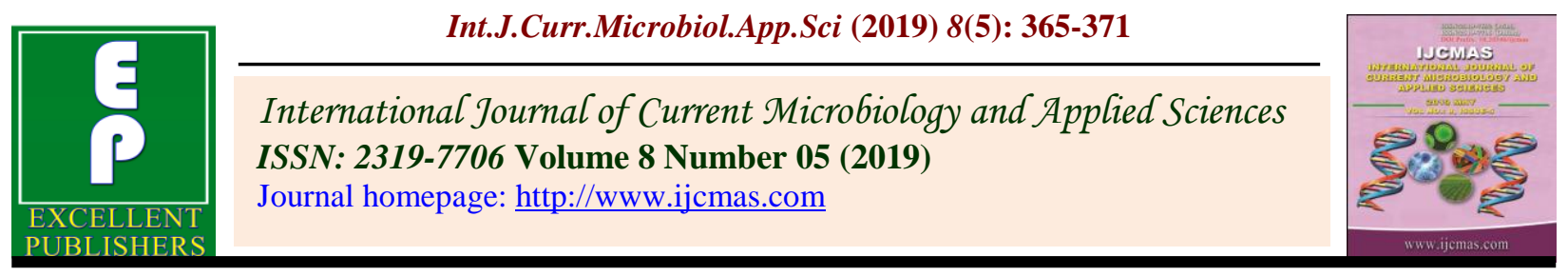

Case Study

https://doi.org/10.20546/ijcmas.2019.805.042

\title{
Management of Heat Stroke in Dogs
}

\author{
P. Abinaya*, A. Abiramy@Prabavathy, D. Selvi, K. Rajkumar, \\ P. Vijayalakshmi and N. Devadevi
}

Department of Veterinary Medicine,Teaching Veterinary Clinical Complex, Rajiv Gandhi Institute of Veterinary Education and Research, Puducherry 605 009, India

*Corresponding author

\section{A B S T R A C T}

\begin{tabular}{|c|c|}
\hline $\begin{array}{l}\text { Ke y w o r d s } \\
\text { Heat stroke, } \\
\text { Dogs, Endotoxemia, } \\
\text { Coagulation } \\
\text { cascade }\end{array}$ & $\begin{array}{l}\text { Heat stress is a form of non fever hyperthermia occurs when heat load exceeds than the } \\
\text { heat loss from the body. The body temperature reaches above } 105-106^{\circ} \mathrm{F} \text {, often } \\
\text { complicated by changes in other body system such as acid-base balance, kidney, liver, } \\
\text { cerebral edema and blood clotting mechanism. More common in brachycephalic breeds, } \\
\text { long thick hairy breeds, young ones and aged animals. Physical examination is marked by }\end{array}$ \\
\hline Article Info & Prevention of heatstroke is achieved primarily by educating owners about proper \\
\hline $\begin{array}{l}\text { Accepted: } \\
\text { 04 April } 2019 \\
\text { Available Online: } \\
10 \text { May } 2019\end{array}$ & $\begin{array}{l}\text { shade and cool water for dogs confined outdoors. The primary goal of treatment is to } \\
\text { lower the body temperature quickly enough to prevent further damage to vital organ } \\
\text { tissues, but not so fast as to cause hypothermia and induce heat-producing mechanisms. }\end{array}$ \\
\hline
\end{tabular}

\section{Introduction}

Heatstroke/hyperthermia is a life-threatening syndrome seen in human beings, dogs and other species. It occurs particularly under humid and hot condition (Coris et al., 2004 and Bruchim et al., 2006). Hyperthermia is the elevation of body temperature due to excessive heat production or absorption, or to deficient heat loss, when the causes of these abnormalities are purely physical. The upper border of the thermo neutral zone - the upper critical temperature - is the effective ambient temperature above which an animal must increase heat loss to maintain thermal balance. The body temperature reaches above $105-106^{\circ} \quad$ F. During hyperthermia, endotoxemia and activation of the coagulation cascade reaction leads to disseminated intravascular coagulation (DIC) and multiorgan dysfunction (MOD) the organs most commonly affected are the brain, kidney, liver, heart and skeletal muscle (Bruchim et al., 2006). Heat stroke is often complicated by acid-base imbalance, cerebral edema and blood clotting mechanism. Heat stroke requires early detection, aggressive therapeutic intervention and continuous critical care monitoring to avoid any secondary complications and death. Heatstroke is characterized by two components, namely (1) elevated core body 
temperature and (2) CNS abnormalities and dysfunctions (Bosak, 2004).

\section{Causes}

Excessive environmental heat and humidity. A rise in body temperature happens when the ambient temperature increases above $86^{\circ} \mathrm{F}$. Break down of the animal's thermal equilibrium occurs when the body temperature reaches $104^{\circ} \mathrm{F}$. At $106^{\circ} \mathrm{F}$ the brain becomes involved and permanent damage may develop.

Dogs that are chained outdoors are commonly affected due to non availability of drinking water and shade. Due to this the animal becomes excited, which further induce the heat stroke. Heat stroke is rare in dogs which are left free regardless of exercise and environmental temperature. Poor ventilation of dog house and pets left in locked cars are the most critical factor for development of heat stroke (Larson and Carithers, 1983).

Neurogenic hyperthermia due to damage in hypothalamus.

Upper airway disease, paralysis of larynx, heart and/or blood vessel disease, nervous system and/or muscular disease and previous history of heat-related disease

Poisoning; some poisonous compounds, such as strychnine and slug and snail bait, can lead to seizures, which can cause an abnormal increase in body temperature

Anesthesia complications

Excessive exercise

\section{Pathogenesis}

Heatstroke may increase the metabolic rate which depletes liver glycogen stores rapidly and further increase in endogenous metabolism of protein. Anorexia is due to respiratory embarrassment and dryness of mouth. Increased heart rate is due to increase in blood temperature, decreased blood pressure and peripheral vasodilation. Increase respiratory rate and depth is due to direct effect on high temperature on respiratory centre, which indirectly cools by increase the salivary secretion, rate of airflow across the respiratory epithelial surface. Oliguria results from decreased renal blood flow and peripheral vasodilation. When the critical temperature is exceeding there is circulatory failure due to myocardial weakness, depression of nervous system activity and respiratory centre. Respiratory failure leads to death. The imbalance between heat generation and dissipation is impacted by thermoregulation, acclimatization, acutephase response (APR), production of heat shock proteins (HSPs), and a patient's predisposing factors (Bouchama and Knochel, 2002).

\section{Predisposing factors}

More common in brachycephalic breeds (short nosed and flat face), long thick hairy breeds, young ones, aged animals, previously affected animals.

Other risk factors are obesity, poor heart / lung conditioning, hyperthyroidisim, insufficient water intake, restricted water diet and dehydration. Forced exercise during times of high environmental temperature.

\section{Clinical signs}

Clinical signs vary based on the duration and degree of exposure to elevated environmental temperature. The clinical signs are panting, increased body temperature $\left(105-107^{\circ} \mathrm{F}\right)$, drooling of saliva, haematemesis, black or tarry colour stools, seizures, muscle tremors, wobbling, incoordination, involuntary paddling, oliguria, turbid and scanty brownish urine (Ettinger and Feldman, 2001). Dark 
urine described as "machine-oil" or "cokecolored" may be present, indicating myoglobinuria (Flournoy et al., 2003). Dehydration, tacchycardia with thready pulse is usually present due to extreme hypovolemia. Pulse deficits may be noted if there is an arrhythmia (Johnson, 1982). Breathing distress, reddened gums, small pinpoint areas of bleeding indicates disseminated intravsacular coagulopathy. Shock, change in mental status and unconsiousness are also noticed. However, it should be pointed out that some animals may have a normal or even subnormal temperature at the time of examination. This occurs especially if the owners have initiated treatment to cool down the animal before presentation or if the patient is in an advanced stage of shock (Drobatz and Macintire, 1976). Clinical presentation can sometimes give clues as to whether the animal's elevated temperature is pyrogenic or nonpyrogenic. Pyrogenic hyperthermia includes infectious and noninfectious systemic inflammatory diseases. Systemic inflammatory diseases characterized by elevated temperature without panting and hypersalivation in dogs. Pyrogenic animals will usually be ambulatory, whereas many heatstroke animals are unwilling or unable to rise (Miller, 2000).

\section{Clinicopathological findings}

\section{Haematological changes}

Hemoconcentration (elevated hematocrit and total solids) associated with dehydration is commonly seen. Low total solids and anemia may be found in some dogs as a result of direct hyperthermic damage, gastrointestinal losses, vasculitis, or renal losses. Thrombocytopenia, prolonged activated partial thromboplastin time, prothrombin time, increased fibrin degradation products, decreased fibrinogen levels, and prolonged activated clotting time can be seen individually or in combination during DIC. Schistocytes may be present on a blood smear, lending support to a presumptive diagnosis of DIC. There may be increased leukocyte numbers; however, severely affected dogs may exhibit marked leukopenia. In addition, blood smears may reveal nucleated red blood cells however, this finding is transient.

\section{Biochemical changes}

Hypoproteinemia; decreased blood glucose concentration is noticed because of increased metabolic demands, hepatic dysfunction or even sepsis. Elevated BUN and creatinine, especially during an acute renal failure crisis. In addition, prerenal factors may contribute to azotemia through dehydration, poor perfusion and hemoconcentration. Hepatocellular damage usually results in elevated liver enzyme concentrations, particularly aspartate transaminase, alanine transaminase and alkaline phosphatase. Mild hyperbilirubinemia may also occur. High levels of creatinine phosphokinase indicate rhabdomyolysis and may reach peak at 24 to 48 hours before declining.

\section{Urine analysis}

In urinalysis increased urine specific gravity, proteinuria, hematuria were observed. Urine sediment should be examined for casts (cellular casts, epithelial cells) indicating renal tubular damage. Myoglobinuria is occasionally noted on urinalysis and indicates rhabdomyolysis.

\section{Electrolyte abnormalities}

Hypernatremia is frequently present due to pure water loss. A mild hyperkalemia may also be present. Hypophosphatemia and hypocalcemia may occur as well, although the mechanism of these changes is unknown. 
Blood gas analysis may reveal respiratory alkalosis reflecting hypocarbia secondary to metabolic acidosis reflecting lactic acid production associated with poor tissue perfusion or excessive muscle activity. Metabolic acidosis in dogs with heatstroke may also be caused by diarrhea or acute renal failure. In addition, mixed acid-base disorders (e.g., respiratory alkalosis, metabolic acidosis) commonly occur. Frequent monitoring of blood gases or total carbon dioxide is recommended during the initial resuscitation of heatstroke patients.

\section{Differential diagnosis}

Heat stroke must be differentiated from eclampsia, hypoglycemia, encephalitis, convulsions, and similar conditions. The sudden onset of signs with high rectal temperatures (excess of $105^{\circ} \mathrm{F}$ ) is usually sufficient for diagnosis (Diehl et al., 2000). Ingestion of tremorgenic toxins such as hexachlorophene, mycotoxins on walnuts or moldy cheese, organophosphates, or metaldehyd has been reported to cause hyperthermia.

\section{Postmortem findings}

The main post-mortem manifestations include (1) diffuse haemorrhagic diathesis, manifested by melena and purpura (2) pulmonary, myocardial and renal bleeding (3) CNS haemorrhages which may result in death (Bruchim et al., 2006). Acute tubular necrosis, pancreatitis and icterus have also been reported. Mild to severe diffuse pulmonary oedema and hyperemia is also noticed. The pulmonary abnormalities included heavy lung, large volumes of frothy exudate in the trachea and bronchi but not in the nasal cavity, blood-stained effusion in pleura. Severe "paint brush" haemorrhages are noticed in the serosal, muscular and submucosal layers throughout the gastrointestinal tract. Moderate splenomegaly and hepatomegaly were also noted in dogs, renal abnormalities included mild to severe bilateral renal swelling and subcapsular haemorrhages, haemorrhages in urinary bladder mucosa, meningeal and brain parenchymal hyperemia and oedema are noticed (Bruchim, 2009).

\section{Treatment}

The immediate step is to reduce the body temperature. Spraying the dog with cool water or immersing the dog in cool - not cold water can be carried out, as this may cause constriction of blood vessels near the surface of the body and may decrease heat dissipation. Total submersion in cold or ice water can cause shock, so one should douse legs, belly, nose, and neck with cool water. The dog's extremities should be rubbed during cooling to promote peripheral circulation. Gradual cooling is always best (Lewis, 1970). Cool the dog with evaporative cooling (such as isopropyl alcohol on, groin, under the forelegs and foot pads) or fan. Allow the dog to drink cool water freely. If possible, the dog should be sprayed down by the owner before being transported to the veterinary hospital. Evaporation can be enhanced by driving with the windows open or placing the dog by the air conditioning vent as this will help with convective heat dissipation. The rectal temperature should be monitored every ten minutes. When the temperature reaches $103^{\circ} \mathrm{F}$ stop cooling with water and wrap the animal with wet towel. Oxygen supplementation via mask, cage or nasal catheter may be used for severe dyspoenic patients, or a surgical opening into the windpipe or trachea may be required if upper airway obstruction is an underlying cause or a contributing factor. After recovery affected animals should always be maintained in a cool $\left(70^{\circ} \mathrm{F}\right)$ oxygen chamber for 24 hours. A systemic, broadspectrum, bacteriocidal antibiotic is often 
administered on the assumption that patients are predisposed to infection. Crystalloid fluids (e.g., balanced electrolyte solutions) are usually the initial fluids of choice because of the need to rehydrate the interstitium. Colloids (e.g., hetastarch, dextran, plasma) may be used during the initial resuscitation period followed by crystalloid therapy shortly thereafter. When both types of fluids are used together, the dose of crystalloids should be reduced $40 \%$ to $60 \%$ (Kirby and Rudloff, 1997).

If peripheral circulatory failure, hemoconcentration, shock or DIC are noticed lactated Ringer's solution are the fluid of choice administered at a rate of $90 \mathrm{~mL} / \mathrm{kg}$ $\mathrm{BW} / \mathrm{hr}$ which corrects dehydration and associated hypovolemia (Tilley and Smith, 2000). Calcium gluconate for possible hypocalcemia resulting from hyperventilation and alkalosis can be administered. Dexamethasone @ 1.0-2.0 mg/kg body weight can be administered to treat shock and potential cerebral edema. However, treatment with corticosteroids is controversial, as they may induce gastrointestinal ulceration, immune suppression and exacerbation of heatstress induced renal damage. However, corticosteroids (prednisolone sodium succinate, dexamethasone sodium phosphate) may prevent cerebral edema and stabilize membranes in some patients experiencing shock. Studies demonstrated that glucocorticoid administration reduced interleukin-1 concentrations and resulted in neuroprotective effects in rats with heatstroke (Liu et al, 2000). NSAIDs, such as dipyrone and flunixin meglumine are contraindicated for heatstroke because they can cause severe hypothermia, gastrointestinal ulceration, prolonged bleeding time, and bone marrow suppression (Mathews, 2000). Mannitol 2.0 $\mathrm{g} / \mathrm{kg}$ body weight as a $20 \%$ solution over a 10 minute period may be used if the patient is stuporous or comatose but should be administered cautiously if DIC is suspected. Gastric and peritoneal lavage with a cold solution. If mean blood pressure falls below $60 \mathrm{~mm} \mathrm{Hg}$ after fluid resuscitation, dopamine or dobutamine hydrochloride should be considered. Doses should be adjusted according to blood pressure monitoring and clinical response. Septic shock should be considered if the animal remains hypotensive after fluid resuscitation. To protect the GI tract $\mathrm{H}_{2}$ blocker, such as cimetidine or ranitidine, may be given one hour after administration of sucralfate. Administration of oral medications in debilitated patients with potentially compromised swallowing reflexes may cause aspiration and therefore, should be used with caution (Fig. 1 and 2).

Fig.1 Petechation noticed in the inguinal region of animal

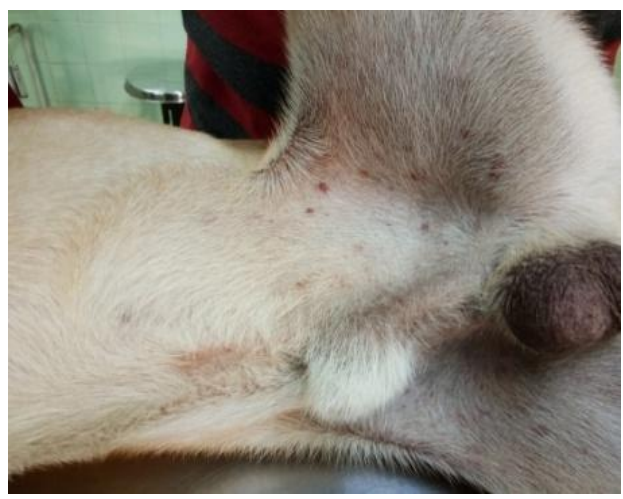


Fig.2and 3 Cooling of animal body

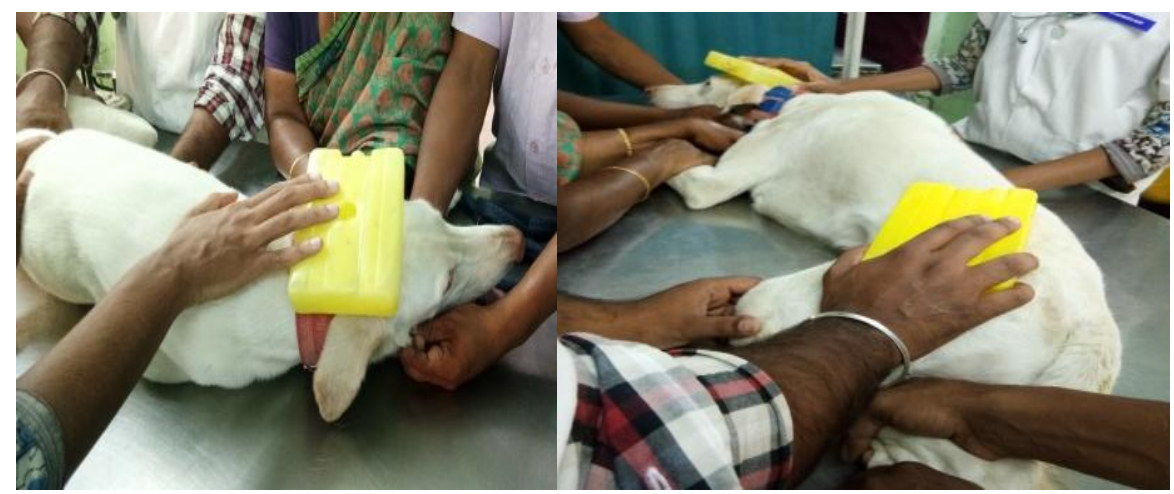

\section{Poor prognostic indicators are}

1. Animals presented comatosed or show progressive neurologic deterioration

2. Hypothermia on presentation

3. Persistent hypoglycemia

4. Worsening azotemia and oliguria despite adequate fluid therapy

5. Evidence of DIC

6. Refractory hypotension

7. Elevated total bilirubin

8. Ventricular arrhythmia

9. Persistent hypoproteinemia

10. Labored respiration, pulmonary edema

\section{Prevention}

Avoid taking your dog out during the peak temperatures of the day, or leaving the dog in places that can become too hot for your dog like a garage, sunny room, sunny yard or car. Never leave your dog in a parked car, even for only a few minutes, as a closed car becomes dangerously hot very rapidly. Always have water accessible to your dog. On hot days provide ice blocks for your dog to lick. If your dog is old or is a brachycephalic breed it needs special attention. Educating the public and clients about the dangers of exercising and confining pets in environments is conducive to heatstroke. Preconditioning pets is necessary. (i.e., exercising regularly and starting out slowly in warm and humid weather until acclimatization occurs).

\section{References}

Bosak, J.K., (2004). Heat stroke in a Great Pyrenees dog. The Canadian Veterinary Journal. 45(6): 513.

Bouchama, A. and Knochel, J. P. (2002). Heat stroke. New England Journal of Medicine, 346: 1978 - 1988.

Bruchim, Y., Klement, E., Saragusty, J., Finkeilstein, E., Kass, P. and Aroch, I. (2006). Heat stroke in dogs: a retrospective study of 54 cases (1999 2004) and analysis of risk factors for death. Journal of Veterinary Internal Medicine, $20: 38$ - 46.

Bruchim, Y., Loeb, E., Saragusty, J. and Aroch, I. (2009). Pathological findings in dogs with fatal heatstroke. Journal of comparative pathology, 140(2-3): 97-104.

Coris, E.E., Ramirez, A. M. and Van Durme, D. J. (2004). Heat illness in athletes: the dangerous combination of heat, humidity and exercise. Sports Medicine, 34: 9 - 16.

Diehl, K.A., Crawford, E., Shinko, P.D., Tallman, R.D. and Oglesbee, M.J. (2000). Alterations in homeostasis associated with hyperthermia in a canine model. Am J Hematol. 64:262270.

Ettinger, S.J., and Feldman, E.C. (2001).Textbook of Veterinary Internal Medicine, 5th ed. 
Philadelphia: WB Saunders:29-30.

Flournoy, S.W., Wohl, J. S. and Macintire, D. K. (2003). Heatstroke in dogs: Pathophysiology and predisposing factors. Compendium on Continuing Education for the Practicing Veterinarian, 25: 410 -418.

Johnson, K.E., (1982). Pathophysiology of heat stroke. Compendium on Continuing Education for the Practicing Veterinarian, 4:141 -145.

Kirby, R., and Rudl off, E. (1997). The critical need for colloids: maintaining fluid balance. The Compendium on continuing education for the practicing veterinarian (USA).

Larson, R.L., and Carithers, R.W. (1983). A review of heat stroke and its complications in the canine. Iowa State University veterinarian. 45(1): 1.

Lewis, S., (1976). Effect of Heat on Canine and Feline. ISU Vet. 38(3):117-121.

Liu, C.C., Chien, C.H. and Lin, M.T.(2000) Glucocorticoids reduce inter-leukin-1 concentration and result in neuroprotective effects in rat heatstroke. J Physiol 527(2):333-343.

Mathews, K., (2000) Nonsteroidal antiinflammatory analgesics. Indications and contraindications for pain management in dogs and ats. Vet Clin North Am Small Anim Pract 30(4):783-804.

Miller, J.B., (2000). Hyperthermia and hypothermia, in Ettinger SJ, Feldman EC (eds): Textbook of Veterinary Internal Medicine, edn. 5. Philadelphia, WB Saunders: 6-10.

Tilley, L.P., and Smith, F.W.K. (2000). The 5-Minute Veterinary Consult, 2nd ed. Philadelphia: Lippincott, Williams and Wilkins, Pp. 756-757.

\section{How to cite this article:}

Abinaya, P., A. Abiramy@Prabavathy, D. Selvi, K. Rajkumar, P. Vijayalakshmi and Devadevi, N. 2019. Management of Heat Stroke in Dogs. Int.J.Curr.Microbiol.App.Sci. 8(05): 365-371. doi: https://doi.org/10.20546/ijcmas.2019.805.042 